\title{
Effect of Individualized Continuous Nursing on Quality of Life of Patients with Hypertension
}

\author{
Ying Liu Shuangyan Dong Ling He Lili Zhang \\ The Second Hospital of Jilin University, Changchun, Jilin, 130000, China
}

\begin{abstract}
Objective: To observe and analyze the effect of individualized continuous nursing on the quality of life of patients with hypertension. Methods: Sixty-four patients who came to our hospital for treatment of hypertension between January 2018 and July 2019 were selected. The patients were given different nursing intervention modes, the patients in the experimental group were given individualized continuous care, and the reference group was given conventional care. To analyze and compare the quality of life scores, systolic and diastolic blood pressure, knowledge of the disease, and medications following the doctor's advice when the two groups were reviewed after discharge. Results: Patients in the experimental group had significantly higher quality of life scores, mastery of disease knowledge, and medication in accordance with the doctor's advice, which were significantly higher than those in the reference group, $\mathrm{P}<0.05$; The difference between the two groups was small, $\mathrm{P}>0.05$; the diastolic and systolic blood pressure of the patients in the experimental group were significantly lower than those in the reference group after nursing, $\mathrm{P}<0.05$. Conclusion: Individualized continuous nursing has a better control effect on patients' blood pressure, can also effectively improve the quality of life of patients, and enable patients to better understand the disease of hypertension, which has high clinical promotion and application value.
\end{abstract}

\section{Keywords}

hypertension; quality of life; conventional nursing; individualized continuing nursing

\section{个体化延续护理对高血压患者生存质量应用的效果分析}

\author{
刘影 董双艳 贺玲 张丽丽 \\ 吉林大学第二医院，中国・吉林 长春 130000
}

\section{摘 要}

目的：观察和分析个体化延续护理对高血压患者生存质量的应用效果。方法：选择的是在 2018 年 1 月 2019 年 7 月期间来我 院治疗高血压的 64 例患者, 分别给予患者不同的护理千预模式, 给予实验组患者个体化延续护理, 给予参照组常规护理, 将 两组患者出院后进行复查时的生存质量评分、收缩压和舒张压、对疾病知识的掌握程度以及遵医嘱服药情况进行分析和比较。 结果: 实验组患者的生存质量评分、对疾病知识的掌握评分、遵医嘱服药情况均显著高于参照组患者, $\mathrm{P}<0.05$; 实验组患 者的收缩压和舒张压在护理前与参照组患者之间的差异较小, $\mathrm{P}>0.05$; 实验组患者的舒张压和收缩压在护理后与参照组相比, 实验组明显较低, $\mathrm{P}<0.05$ 。结论: 个体化延续护理对患者的血压起到了较好的控制作用, 还能够有效提升患者的生存质量, 并且使患者更加了解高血压这一疾病, 临床推广和应用价值高。

\section{关键词}

高血压；生存质量；常规护理；个体化延续护理

1 引言

高血压已经成了临床上非常常见的慢性疾病, 患者需要 终身服药来控制血压, 根据相关研究证实, 全球大概有超过 十亿的患者受到高血压疾病的困扰，而高血压会诱发心脑血 管方面的疾病, 因此致残率和致死率都较高, 严重影响了患 者的生存质量 ${ }^{[1-2]}$ 。因此, 选择了来我院接受高血压治疗的 64 例患者进行研究，研究的时间为 2018 年 1 月 2019 年 7 月, 患者被分为两组, 并接受不同的护理干预, 通过进行对比旨
在研究个体化延续护理的临床效果。内容汇报如下。

\section{2 资料与方法}

\section{1 一般资料}

本文选择了 64 例来我院治疗高血压的患者作为对象进 行研究，患者入院的时间范围是 2018 年 1 月 2019 年 7 月。 所有患者通过诊断, 舒张压均超过 $90 \mathrm{mmHg}$ 或收缩压均超过 $140 \mathrm{mmHg}$, 同意参与本次研究, 并与家属共同签署了知情协议。 研究将排除精神异常、存在交流障碍的患者; 患有严重肾功 
表 1 比对两组患者的各项生存质量评分 ( $\mathrm{x} \pm \mathrm{s}$, 分)

\begin{tabular}{|c|c|c|c|c|c|c|c|c|c|}
\hline 组别 & 例数 & 生理功能 & 生理职能 & 社会功能 & 躯体疼痛 & 情感职能 & 活力 & 精神健康 & 总体健康 \\
\hline 实验组 & 32 & $23.45 \pm 1.76$ & $14.38 \pm 5.34$ & $9.65 \pm 1.47$ & $11.02 \pm 1.38$ & $8.34 \pm 1.18$ & $18.64 \pm 3.75$ & $22.47 \pm 3.35$ & $21.24 \pm 3.28$ \\
\hline 参照组 & 32 & $16.37 \pm 5.54$ & $9.18 \pm 2.20$ & $7.30 \pm 3.42$ & $9.18 \pm 2.47$ & $5.49 \pm 1.82$ & $14.15 \pm 2.28$ & $18.76 \pm 2.48$ & $17.65 \pm 3.71$ \\
\hline $\mathrm{t}$ & -- & 6.89 & 5.09 & 3.57 & 3.67 & 7.43 & 5.78 & 5.03 & 4.10 \\
\hline $\mathrm{P}$ & -- & 0.00 & 0.00 & 0.00 & 0.00 & 0.00 & 0.00 & 0.00 & 0.00 \\
\hline
\end{tabular}

能不全、脑卒中、心肌梗死的患者; 临床资料保存不完整或 未来医院复查的患者。6 4 例患者被随机分为两组, 实验组和 参照组。其中有 32 例患者进入实验组内, 年龄范围是 $45 \sim 78$ 岁之间, 年龄均值为 $(62.15 \pm 7.43)$ 岁, 女性和男性的例数 比为 18: 14 , 病程在 1 10 年, 平均病程为 $(5.34 \pm 3.21)$ 年; 另外 32 例患者进入参照组内，年龄范围是 46 77 岁之间，年 龄均值为 $(62.34 \pm 7.62)$ 岁, 女性和男性的例数比为 $17: 15$, 病程在 1 9.5 年, 平均病程为 $(5.28 \pm 3.11)$ 年, 两组患者的 年龄、病程和性别均通过 SPSS 25.0 处理, 结果显示可比。

\section{2 方法}

实施常规护理的是参照组患者，主要包括用药指导，指 导患者家属为患者测量血压, 为患者发放健康教育手册, 进行 健康宣教。

实施个体化延续性护理的是实验组, 主要内容有以下几 点: (1) 建立护理小组。有护士长和三名有丰富护理经验的 护理人员共同组建个体化延续护理小组，定期对其他护理人 员开展培训和考核, 提升护理人员的护理质量, 要求护理人 员掌握高血压疾病护理的相关知识。（2）制定护理计划。护 理小组要对患者的病情、家庭情况、认知情况等进行评估， 之后共同讨论为患者制定具有个性化的延续护理方案, 并选 择责任护士。（3 ）建立健康档案。护理人员要为每一位患者 建立健康档案，包括患者过敏史、禁忌症、治疗过程、家庭 地址、电话号码等基本情况, 并将护理情况详细记录。（4） 电话随访。在患者出院之后, 护理人员每周都要给患者或其 家属打电话, 了解患者是否掌握了有关高血压疾病的知识, 并了解患者的用药情况、血压自测情况, 根据了解的情况给 予患者必要的指导, 以提升治疗效果和患者的依存性。（5) 家庭随访。在患者出院, 护理人员每隔一个月就要到患者的 家庭了解情况, 掌握患者在家庭中的生活情况, 并主动询问 患者有无出现不良反应, 叮嘱患者及其家属如果出现不良反 应, 则需要及时与主治医师取得联系; 护理人员也要了解患 者的生活习惯和饮食习惯，给予患者具体的指导，以帮助患
者养成良好习惯。（ 6 ）定期复诊。护理人员要告知患者家属, 要定期来医院或门诊进行复诊，接受常规的检查。

\section{3 观察指标}

(1) 将两组患者出院后进行复查时的生存质量评分进行 观察和分析, 用 SF-36生活质量量表进行评价, 包括生理功能、 生理职能、社会功能、情感职能、躯体疼痛、活力、精神健 康以及总体健康, 分数越高表示患者生活质量越好 ${ }^{[3]}$ 。 观察和分析两组患者在护理前后收缩压和舒张压的变化情况。 (3) 观察患者对疾病知识的掌握程度 (本院自制调查问卷, 调查患者对高血压相关知识的掌握情况）和遵医嘱服药情况 ( 遵医行为: 可以长期进行规范服药并且积极接受治疗为完 全遵医; 偶发发生忘记服药或出现不规范服药情况为部分遵 医；无法坚持或经常中断服药为不遵医）。

\section{4 统计学处理}

实验组和参照组患者与本次研究相关的数据和基本资料 都通过 SPSS 25.0 进行分析, 生存质量评分、护理前后收缩 压和舒张压、知识掌握程度均用 $(` \mathrm{x} \pm \mathrm{s}$ ) 表示, $\mathrm{t}$ 检验, $\mathrm{P}<0.05$; 遵医行为用 $(\mathrm{n} / \%)$ 表示, $\mathrm{X}^{2}$ 检验， $\mathrm{P}<0.05$ 。

\section{3 结果}

\section{1 比对两组患者的各项生存质量评分}

由表 1 显示, 实验组患者的各项生存质量均显著优于参 照组, $\mathrm{P}<0.05$ 。

\section{2 比对两组患者治疗前后的收缩压和舒张压}

由表 2 显示, 护理前, 两组患者的血压指标之间无显著 差异, $\mathrm{P}>0.05$; 护理后, 实验组患者血压指标显著优于参照 组, $P<0.05$ 。

表 2 比对两组患者治疗前后的收缩压和舒张压 ( ${ }^{x} \pm \mathrm{s}, \mathrm{mmHg}$ )

\begin{tabular}{|c|c|c|c|c|c|}
\hline \multirow{2}{*}{ 组别 } & \multirow{2}{*}{ 例数 } & \multicolumn{2}{|c|}{ 舒张压 } & \multicolumn{2}{c|}{ 收缩压 } \\
\cline { 3 - 6 } 实验组 & 32 & $97.2 \pm 6.3$ & $82.4 \pm 4.8$ & $148.2 \pm 8.5$ & $123.6 \pm 7.1$ \\
\hline 参照组 & 32 & $97.5 \pm 6.1$ & $89.4 \pm 5.5$ & $148.4 \pm 8.6$ & $141.5 \pm 5.8$ \\
\hline $\mathrm{t}$ & -- & 0.19 & 5.42 & 0.09 & 11.04 \\
\hline $\mathrm{P}$ & -- & 0.84 & 0.00 & 0.92 & 0.00 \\
\hline
\end{tabular}




\section{3 比对两组患者的遵医率和知识掌握程度}

由表 3 显示, 实验组患者的遵医率以及知识掌握评分都 明显高于参照组， $\mathrm{P}<0.05$ 。

表 3 比对两组患者的遵医率和知识掌握程度（ $\mathrm{x} \pm \mathrm{s} ％$ )

\begin{tabular}{|c|c|c|c|c|c|c|}
\hline \multirow[b]{2}{*}{ 组别 } & \multirow[b]{2}{*}{ 例数 } & \multicolumn{4}{|c|}{ 遵遵医行为 } & \multirow{2}{*}{$\begin{array}{c}\text { 知识掌握评 } \\
\text { 分 }\end{array}$} \\
\hline & & $\begin{array}{c}\text { 完全遵 } \\
\text { 医 }\end{array}$ & $\begin{array}{c}\text { 一般遵 } \\
\text { 医 }\end{array}$ & 不遵医 & 遵医率 & \\
\hline 实验组 & 32 & 26 & 4 & 0 & $32(100.00)$ & $82.63 \pm 5.76$ \\
\hline 参照组 & 32 & 15 & 8 & 9 & $23(71.88)$ & $72.98 \pm 7.68$ \\
\hline $\mathrm{t}$ & -- & \multicolumn{4}{|c|}{10.47} & 5.68 \\
\hline $\mathrm{P}$ & -- & \multicolumn{4}{|c|}{0.00} & 0.00 \\
\hline
\end{tabular}

\section{4 讨论}

高血压一经确诊, 就需要终身服药, 容易引发脑卒中、 脑出血、心肌梗死等心脑血管疾病，并且随着患者年龄的逐 渐上升，患该病的概率也会逐渐增大。患者在早期并无明显 表现，随着病情的发展，患者会出现头痛、恶心等症状，降 低了其生活质量 ${ }^{[4]}$ 。有研究显示, 如果良好的控制患者的血压, 则发生心脑血管疾病的概率也会有所降低，患者的生存质量 就会得到提升 ${ }^{[5]}$ 。要想控制患者的血压，日常的药物治疗远 远不够, 还需要采取相应的措施来提升患者的依存性和遵医 嘱率。

本次研究给予实验组患者个体化延续性护理，各项生存 质量评分相比于参照组, 实验组明显较高, $\mathrm{P}<0.05$; 护理后, 实验组的舒张压和收缩压为 $(82.4 \pm 4.8)$ 、 $(89.4 \pm 5.5)$, 而参照组的分别是 $(123.6 \pm 7.1) 、(141.5 \pm 5.8)$, 实验组 的血压下降明显, 并且存在优势, $\mathrm{P}$ 下余 0.05 ; 实验组的遵医 率为 $100.00 \%$ 。而参照组仅仅有 $71.88 \%$ ，实验组知识掌握评 分为 $(82.63 \pm 5.76)$, 参照组为 $(72.98 \pm 7.68)$, 实验组在 遵医率和知识掌握评分方面都显著高于参照组, $\mathrm{P}<0.05$ 。究 其原因: 个体化延续护理主要是根据患者的具体病情为其制
定个性化的护理方案, 之后严格实施, 通过随访和复诊, 对 患者的病情进行护理, 根据患者的具体情况为其制定护理方 案, 能更加具有针对性, 提升了治疗效果, 使得患者的血压 能得到更好的控制; 同时针对患者的治疗情况, 进行随访和 复查, 给予患者必要的指导, 并教患者自我护理的方法, 提 升患者对知识的认知程度, 让每一位患者都能认识高血压, 掌握高血压的基本知识, 提高患者的依存性和护理满意度 ${ }^{[6-7]}$ 。

\section{5 结语}

综上所述，个体化延续护理患者的对高血压患者实施个 体化延续护理, 能够改善患者的生活质量, 提升其对知识的 认知程度，可以在临床上广泛应用。

\section{参考文献}

[1] 刘玉英. 个体化延续护理对改善高血压患者生存质量的效果评价 [J]. 中国妇幼健康研究 ,2017,28(S1):225-226.

[2] 李晓慧, LIXiaohui. 个体化延续护理对老年高血压患者血压控制及 不良事件预防效果研究 [J]. 实用临床医药杂志 ,2017,21(4):14.

[3] 陈雄虎, 王莹莹, 李桂兰. 阶段性延续护理对高血压脑出血患者生 活质量的影响 [J]. 上海护理 ,2017,17(4):27-29.

[4] 热孜万古丽 \&\#; 艾合买提, 郭英, 张辉, 等. 延续护理对高血压患 者血压、体质指数的影响 [J]. 西部中医药, 2018,31(3):124-126.

[5] 陈红梅. 超早期康复护理对高血压脑出血病人预后的影响 [J]. 安 徽医药 ,2019,23(9): 1789-1792.

[6] 冷雅楠, 李玉琦, 刘婷婷, 等. 老年高血压患者衰弱发生率及影 响因素的 Meta 分析 [J]. 中华现代护理杂志, 2019,25(20):2545-2550.

[7] 蒲卫虹 [1], 张琴 [2], 何小玉 [1], 等. 基于人性化理念的心理护理 对高血压脑出血术后患者焦虑状态和满意度的影响 [J]. 国际精神 病学杂志 ,2017,44(3):546-548 